\title{
A CpG island methylator phenotype of colorectal cancer that is contiguous with conventional adenomas, but not serrated polyps
}

\author{
KOJI HOKAZONO ${ }^{1}$, TAKASHI UEKI ${ }^{1}$, KINUKO NAGAYOSHI ${ }^{1}$, YASUNOBU NISHIOKA ${ }^{1}$, TATSUNOBU HATAE ${ }^{1}$, \\ YUTAKA KOGA $^{2}$, MINAKO HIRAHASHI ${ }^{2}$, YOSHINAO ODA ${ }^{2}$ and MASAO TANAKA ${ }^{1}$
}

Departments of ${ }^{1}$ Surgery and Oncology, and ${ }^{2}$ Anatomical Pathology, Graduate School of Medical Sciences, Kyushu University, Fukuoka, Fukuoka 812-8582, Japan

Received January 28, 2014; Accepted July 15, 2014

DOI: $10.3892 / 01.2014 .2430$

\begin{abstract}
A subset of colorectal cancers (CRCs) harbor the $\mathrm{CpG}$ island methylator phenotype (CIMP), with concurrent multiple promoter hypermethylation of tumor-related genes. A serrated pathway in which CIMP is developed from serrated polyps is proposed. The present study characterized CIMP and morphologically examined precursor lesions of CIMP. In total, 104 CRCs treated between January 1996 and December 2004 were examined. Aberrant promoter methylation of 15 cancer-related genes was analyzed. CIMP status was classified according to the number of methylated genes and was correlated with the clinicopathological features, including the concomitant polyps in and around the tumors. The frequency of aberrant methylation in each CRC showed a bimodal pattern, and the CRCs were classified as CIMP-high (CIMP-H), CIMP-low (CIMP-L) and CIMP-negative (CIMP-N). CIMP-H was associated with aberrant methylation of $M L H 1(\mathrm{P}=0.005)$ and with an improved recurrence-free survival (RFS) rate following curative resection compared with CIMP-L/N (five-year RFS rate, 93.8 vs. 67.1\%; P=0.044), while CIMP-N tumors were associated with frequent distant metastases at diagnosis $(\mathrm{P}=0.023)$. No concomitant serrated lesions were present in the tumors, whereas conventional adenoma was contiguous with $11(10.6 \%)$ of 104 CRCs, including four CIMP-H CRCs. CIMP-H was classified in CRCs by a novel CIMP marker panel and the presence of concomitant tumors revealed that certain CIMP-H CRCs may have arisen from conventional adenomas.
\end{abstract}

\section{Introduction}

The presence of the $\mathrm{CpG}$ island (CGI) methylator phenotype (CIMP) in colorectal cancers (CRCs) has been supported by

Correspondence to: Dr Takashi Ueki, Department of Surgery and Oncology, Graduate School of Medical Sciences, Kyushu University, 3-1-1 Maidashi, Higashi-ku, Fukuoka, Fukuoka 812-8582, Japan E-mail: tueki@surg1.med.kyushu-u.ac.jp

Key words: $\mathrm{CpG}$ island methylator phenotype, colorectal cancer, methylation, promoter $\mathrm{CpG}$ island, serrated pathway the fact that one group of CRCs has few methylated promoter CGIs and another group harbors simultaneous aberrant methylation of multiple promoter CGIs $(1,2)$. CIMP-positive CRCs have distinct clinical and histological features, including a female predominance and proximal location, and show genetic characteristics, including frequent $K R A S / B R A F$ mutations and microsatellite instability (MSI) $(3,4)$. CIMP is initially defined using cancer-specific CIMP markers (CDKN2A, MINT1, MINT2, MINT31 and MLH1) in CRCs (2), but in 2006, Weisenberger et al (5) challenged the application of these classic CIMP markers and insisted upon the efficacy of novel marker panels to endorse the CIMP as a distinctive molecular feature of CRCs. Although based on a systematic analysis of a large number of CRCs with aberrant methylation of numerous promoter CGIs, later studies failed to emulate the original results using the same markers selected by Weisenberger et al $(4,6)$. No matter how the markers are selected, CIMP is certain to be involved in CRC development as the third molecular pathway, following chromosomal instability and MSI. Ogino et al showed that CIMP-positive $\mathrm{CRC}$ was a predictor of a low cancer-specific mortality rate in a large cohort study (4). By contrast, using different CIMP marker panels, this characteristic of CIMP-positivity was not observed in patients with stage III CRCs (7) or stage II/III CRCs treated by surgery alone (8). The response to 5-fluorouracil (5-FU)-based adjuvant chemotherapy in CIMP-positive CRC is also contradictory (9-11), although this therapy is essential to reduce the tumor recurrence of stage II or III CRC patients following curative resection.

Hypermethylation of promoter CGIs can prevent transcription of tumor suppressor or mismatch repair genes, such as MutL homolog 1 (MLH1), and occurs at an early stage of colorectal carcinogenesis. Methylation of promoter CGIs followed by transcriptional silencing of $M L H 1$ is present in $\sim 70 \%$ of sporadic MSI CRCs $(8,12,13)$. However, $M L H 1$ is usually included in CIMP marker sets of promoter CGIs, and up to $60 \%$ of CIMP-positive CRCs have aberrant methylation of $M L H 1$ (14). This may be one of the reasons for the clinical and pathological resemblance between CIMP-positive and MSI CRCs. The high frequency of serrated polyps with $M L H 1$ gene promoter methylation in individuals with MSI CRC suggests the presence of a serrated pathway in colorectal carcinogenesis (15). More recently, genetic and epigenetic profiles 
of a variety of colorectal polyps have demonstrated that sessile serrated adenomas/polyps may be precursor lesions for MSI CRCs and follow the CIMP pathway (16). Since a considerable fraction of advanced CRCs in the adenoma-adenocarcinoma sequence had a remnant adenomatous element within the tumors and coexisting extralesional adenomas (17), it is important to examine whether CIMP-positive CRCs have similar morphological characteristics regarding serrated polyps.

In the present study, a series of CRCs were retrospectively examined for aberrant methylation using an alternative panel of promoter CGIs of cancer-related genes. The panel consisted of promoter CGIs of tumor suppressor genes (p16, GATA5, TSLC1, HLTF and ID4), DNA repair genes (MGMT), metastases suppressor genes (TIMP3, CDH4 and $C D H 13)$, angiogenesis inhibitors (TSP1) and genes with apoptosis-related properties (HRK, CACNAIG and RSASF1A). The majority of these genes are not involved as classical or novel CIMP markers of CRC $(2,5,14)$. The panel also contained two novel genes that were originally cloned in pancreatic cancer, which were methylated in the cancer cells, but not in the normal pancreas or colonic mucosa (18). The CIMP was defined by comparing the observed distribution of CRCs by the number of aberrant methylations of these genes with the calculated distribution. The CIMP status was correlated with the methylation status of $M L H 1$ and with clinicopathological parameters, with particular reference to neighboring lesions, such as conventional adenoma and serrated lesions, in and around tumors.

\section{Materials and methods}

Patient population and DNA preparation. Neoplastic specimens were collected from consecutive patients who underwent CRC resection at Kyushu University Hospital (Fukuoka, Japan) between 1996 and 2004. From these tumor specimens, 104 CRC frozen tissues were used and the frozen tissue of 15 corresponding normal mucosae were also collected. Clinical data and the patient status at the last follow-up were obtained from medical records. Informed consent to harvest the tissue for the studies was obtained from all patients, and the Kyushu University Hospital Human Research Ethics Committee approved the study. Genomic DNA was prepared from cryostat sections of the frozen cancer tissue and corresponding normal mucosa specimens and was extracted by QIAamp DNA Mini kit (Qiagen, Hilden, Germany). Hematoxylin and eosin-stained sections of formalin-fixed and paraffin-embedded surgical specimens were evaluated to determine tumor differentiation and stage. All polyps present in the specimen were also sectioned and prepared for histological examination.

Bisulfite modification and methylation-specific polymerase chain reaction (MSP) assay. The methylation status of each gene was verified by MSP, as described by Herman et al (19). Genomic DNA from the cancer tissue and the normal mucosa was treated with bisulfite for $16 \mathrm{~h}$ at $50^{\circ} \mathrm{C}$ to convert unmethylated cytosine to thymine. Polymerase chain reaction (PCR) primers for each gene were designed to be specific for the methylated sequence and the promoter region of each gene. Three to six $\mathrm{CpG}$ sites were included in each primer pair to achieve optimal specificity for the determination of methylation. MSP was carried out on $1 \mu \mathrm{l}$ bisulfite-treated DNA with the following amplification conditions: $95^{\circ} \mathrm{C}$ for 5 min, followed by 40 cycles of $94^{\circ} \mathrm{C}$ for $30 \mathrm{sec}$, annealing for $30 \mathrm{sec}$ and $72^{\circ} \mathrm{C}$ for $30 \mathrm{sec}$, with a final extension at $72^{\circ} \mathrm{C}$ for 5 min. All PCRs were performed with CpGenome Universal Methylated DNA (Chemicon International, Temecula, CA, USA) as a positive control for methylated alleles and with no DNA as a negative control. The primer sequences and the specific annealing temperatures for the 15 genes and $\mathrm{MLH1}$ are shown in Table I. PCR products $(5 \mu \mathrm{l})$ were separated by $3 \%$ agarose gel electrophoresis and visualized under ultraviolet illumination, following ethidium bromide staining. The presence of PCR products indicated the presence of methylated template sequences in the original genomic DNA.

Statistical analysis. The primary variable in this study was the distribution of carcinomas falling into each classification of the number of aberrantly methylated genes. The observed distribution of the 104 CRCs was compared with the expected distribution by $\chi^{2}$ test (goodness-of-fit test) under the assumptions that promoter methylation of each gene occurred randomly and was distributed equally in the carcinomas. The association between CIMP status and clinicopathological parameters was assessed by Fisher's exact test, analysis of variance or Mann-Whitney U test. Event time distributions for overall survival (OS) and relapse-free survival (RFS) of our 104 CRC patients were estimated with the Kaplan-Meier method. Hazard ratios (HRs) of tumor-relapse, according to the clinicopathological features and the CIMP status in tumors, were analyzed by Cox proportional hazard models. All $\mathrm{P}$-values were two-sided, and $\mathrm{P}<0.05$ was considered to indicate a statistically significant difference.

\section{Results}

Classification of CIMP and methylation of each gene in CRCs. The number of aberrantly methylated genes in each $\mathrm{CRC}$ ranged between zero and 14 . The expected distribution of carcinomas having each number of aberrantly methylated genes among 104 CRCs was calculated, assuming that hypermethylation of the 15 genes occurred independently and was spread randomly across the $104 \mathrm{CRCs}$. The expected distribution followed a unimodal pattern, with the largest number of carcinomas having four methylated genes (Fig. 1, white bars). In the expected distribution, ten CRCs (9.6\% of the $104 \mathrm{CRCs}$ ) were predicted to have seven or more methylated genes, and the number of CRCs without any methylated genes was zero (0\% of the $104 \mathrm{CRCs}$ ). The observed distribution of carcinomas having each number of aberrantly methylated genes did not follow the expected distribution (Fig. 1, black bars). Carcinomas with methylation of seven or more of the 15 genes were classified as CIMP-high (CIMP-H), carcinomas with methylation of one to six genes were classified as CIMP-low (CIMP-L) and carcinomas without methylation were classed as CIMP-negative (CIMP-N). There were 19 (18.3\%) CIMP-H CRCs, 76 (73.1\%) CIMP-L CRCs and 9 (8.7\%) CIMP-N CRCs. The observed distribution of methylated genes in each group differed significantly from the expected distribution $(\mathrm{P}<0.001)$, thus, methylation of these genes did not happen at random in the 104 CRCs. Methylation of each gene and the CIMP status 
Table I. Methylated primer sequences and annealing temperatures used in methylation-specific polymerase chain reaction.

\begin{tabular}{|c|c|c|c|c|}
\hline Gene & $\mathrm{F} / \mathrm{R}$ & Sequences & Temperature, ${ }^{\circ} \mathrm{C}$ & Size, bp \\
\hline p16 & $\begin{array}{l}\mathrm{F} \\
\mathrm{R}\end{array}$ & $\begin{array}{l}\text { 5'-TAATAGTATTTTTTTCGAGTATTC-3' } \\
\text { 5'-TTCTTCCTCCGATACTAACG-3' }\end{array}$ & 54 & 123 \\
\hline$h M L H 1$ & $\begin{array}{l}\mathrm{F} \\
\mathrm{R}\end{array}$ & $\begin{array}{l}\text { 5'-TAATAGGAAGAGCGGATAGC-3' } \\
\text { 5'-TCTATAAATTACAAATCTCTTCG-3' }\end{array}$ & 54 & 106 \\
\hline TIMP3 & $\begin{array}{l}\mathrm{F} \\
\mathrm{R}\end{array}$ & $\begin{array}{l}\text { 5'-GGGTCGATGAGGTAATGC-3' } \\
\text { 5'-TACGCCGCTACCTAAACG-3' }\end{array}$ & 64 & 116 \\
\hline$M G M T$ & $\begin{array}{l}\mathrm{F} \\
\mathrm{R}\end{array}$ & $\begin{array}{l}\text { 5'-GTTTTAGATTTCGTTTTACGTC-3' } \\
\text { 5'-CCAAATCGCAAACGATACG-3' }\end{array}$ & 54 & 145 \\
\hline$T S P 1$ & $\begin{array}{l}\mathrm{F} \\
\mathrm{R}\end{array}$ & $\begin{array}{l}\text { 5'-GAAAGTTTTTGCGTTATTTCGC-3' } \\
\text { 5'-CTTAACGCACACGAACTCG-3' }\end{array}$ & 64 & 130 \\
\hline CACNAIG & $\begin{array}{l}\mathrm{F} \\
\mathrm{R}\end{array}$ & $\begin{array}{l}\text { 5'-TTTTAGATTCGGTTTTAGTTGC-3' } \\
\text { 5'-AACTCCGATTACCGAATTCG-3' }\end{array}$ & 54 & 140 \\
\hline $\mathrm{CDH} 4$ & $\begin{array}{l}\mathrm{F} \\
\mathrm{R}\end{array}$ & $\begin{array}{l}\text { 5'-GTTTTCGGTGTCGGGTATC-3' } \\
\text { 5'-CGACAACTTACCCGAAACG-3' }\end{array}$ & 66 & 105 \\
\hline$H-C A D$ & $\begin{array}{l}\mathrm{F} \\
\mathrm{R}\end{array}$ & $\begin{array}{l}\text { 5'-TTCGCGGGGTTCGTTTTTC-3' } \\
\text { 5'-AATAAATCAACAACAACATCACG-3' }\end{array}$ & 67 & 147 \\
\hline GATA5 & $\begin{array}{l}\mathrm{F} \\
\mathrm{R}\end{array}$ & $\begin{array}{l}\text { 5'-TTCGGGTCGTTGAGGTTTC-3' } \\
\text { 5'-CAAAATCACGTAACTCTACG-3' }\end{array}$ & 64 & 140 \\
\hline RASSF $1 A$ & $\begin{array}{l}\mathrm{F} \\
\mathrm{R}\end{array}$ & $\begin{array}{l}\text { 5'-CGAGAGCGCGTTTAGTTTC-3' } \\
\text { 5'-CAAAATCCAAACTAAACGACG-3' }\end{array}$ & 58 & 103 \\
\hline HLTF & $\begin{array}{l}\mathrm{F} \\
\mathrm{R}\end{array}$ & $\begin{array}{l}\text { 5'-CGTTTCGTTGTTATTTAAAGAC-3' } \\
\text { 5'-CCGCAAACACCGCAATCG-3' }\end{array}$ & 60 & 132 \\
\hline$H R K$ & $\begin{array}{l}\mathrm{F} \\
\mathrm{R}\end{array}$ & $\begin{array}{l}\text { 5'-AATTTCGCGTTTTTTAGTTGTC-3' } \\
\text { 5'-GAAAAAAAAAATTACATCATCCG-3' }\end{array}$ & 54 & 115 \\
\hline KIRREL2 & $\begin{array}{l}\mathrm{F} \\
\mathrm{R}\end{array}$ & $\begin{array}{l}\text { 5'-TTGGGGGCGTTTATTCGTC-3' } \\
\text { 5'-GCCCCCCGAAAACTCCG-3' }\end{array}$ & 62 & 105 \\
\hline SLC13A5 & $\begin{array}{l}\mathrm{F} \\
\mathrm{R}\end{array}$ & $\begin{array}{l}\text { 5'-GTTTAGCGTCGAGGTTATC-3' } \\
\text { 5'-TACGAAACGAAATTATCACCG-3' }\end{array}$ & 67 & 137 \\
\hline ID4 & $\begin{array}{l}\mathrm{F} \\
\mathrm{R}\end{array}$ & $\begin{array}{l}\text { 5'-ATTTTTCGTTTTTTAGTATCGTTC-3' } \\
\text { 5'-ACGCGCGAACCGAATCG-3' }\end{array}$ & 62 & 104 \\
\hline TSLC1 & $\begin{array}{l}\mathrm{F} \\
\mathrm{R}\end{array}$ & $\begin{array}{l}\text { 5'-TAATCGTTGTATTAGATCGAC-3' } \\
\text { 5'-TAAATTTACAACGTCTAATTCG-3' }\end{array}$ & 60 & 103 \\
\hline
\end{tabular}

F, forward primers; R, reverse primers; $h M L H 1$, human MutL homolog 1.

are summarized in Fig. 2. The frequency of hypermethylation of the 15 genes in the 104 CRCs ranged from $5.8 \%(H R K)$ to $77.9 \%(\mathrm{CDH} 4)$, whereas methylated templates were not detected in the 15 normal colonic mucosae.

CIMP status, clinicopathological parameters and MLHI methylation. The association between CIMP status, the clinicopathological features and $M L H 1$ methylation are shown in Table II. There was no significant correlation between the CIMP status and the parameters among the 104 patients with respect to age, gender, tumor size, histological tumor grade or tumor stage. In total, 12 patients exhibited distant metastases, the majority of which were liver metastases, and three patients presented with peritoneal metastases at diagnosis. MLH1 methylation was detected in 10 (9.6\%) of 104 CRCs. Six of these were classified as CIMP-H (31.6\% of 19 CIMP-H tumors) and four were CIMP-L (5.3\% of 76 CIMP-L tumors), but none of the nine CIMP-N tumors exhibited $\mathrm{MLH} 1$ methylation $(\mathrm{P}=0.005)$. The patients with CIMP-N CRCs had more frequent distant metastases compared with those with CIMP-H/L tumors $(44.4,15.8$ and $10.5 \%$, respectively, $\mathrm{P}=0.023)$. 5-FU-based chemotherapy was post-operatively performed in $63.2 \%$ of patients with CIMP-H CRCs, $64.5 \%$ of those with CIMP-L and $77.8 \%$ of those with CIMP-N. Within the median follow-up time of 60 months, two (40.0\%) out of five patients with stage 0-III CIMP-N and 22 (32.4\%) out of 68 patients with stage 0-III CIMP-L developed tumor recurrence following curative resection, while only one $(6.3 \%)$ out of 16 patients with stage 0-III CIMP-H tumors developed recurrence $(\mathrm{P}=0.093)$.

The rate of tumor recurrence in the CIMP-N and CIMP-L tumors was similar; therefore, 104 CRCs were divided into two groups, CIMP-L/N and CIMP-H, for further survival time analysis. Kaplan-Meier survival curves representing the OS rates of all patients and the RFS rate of 89 patients with stage 0 -III tumors, according to CIMP status, are shown 
Table II. Association between the CIMP and the clinicopathological features of 104 colorectal cancers.

\begin{tabular}{|c|c|c|c|c|c|}
\hline Features & Total & CIMP-N & CIMP-L & CIMP-H & P-value \\
\hline No. of patients & $104(100.0)$ & $9(8.7)$ & $76(73.1)$ & $19(18.3)$ & \\
\hline Mean age, years & 63.4 & 60.7 & 62.9 & 66.6 & 0.317 \\
\hline \multicolumn{6}{|l|}{ Gender, n (\%) } \\
\hline Male & $51(49.0)$ & $5(55.6)$ & $35(46.1)$ & $10(52.6)$ & \\
\hline Female & $53(51.0)$ & $4(44.4)$ & $41(53.8)$ & $9(47.4)$ & 0.471 \\
\hline \multicolumn{6}{|l|}{ Tumor location, n (\%) } \\
\hline Proximal & $42(40.4)$ & $1(11.1)$ & $32(42.1)$ & $9(47.4)$ & \\
\hline Distal & $62(59.6)$ & $8(88.9)$ & $44(57.9)$ & $10(52.6)$ & 0.118 \\
\hline Mean tumour size, mm & 48.2 & 47.7 & 49.7 & 43.7 & 0.466 \\
\hline \multicolumn{6}{|l|}{ Histology, n (\%) } \\
\hline Differentiated & $90(86.5)$ & $7(77.8)$ & $67(91.3)$ & $16(84.2)$ & \\
\hline Undifferentiated & $14(13.5)$ & $2(22.2)$ & $9(8.7)$ & $3(15.8)$ & 0.680 \\
\hline \multicolumn{6}{|l|}{ Lymphatic invasion, n (\%) } \\
\hline Negative & $51(49.0)$ & $5(55.6)$ & $38(50.0)$ & $8(42.1)$ & \\
\hline Positive & $53(51.0)$ & $4(44.4)$ & $38(50.0)$ & $11(58.9)$ & 0.760 \\
\hline \multicolumn{6}{|l|}{ Venous invasion, $\mathrm{n}(\%)$} \\
\hline Negative & $66(57.4)$ & $5(55.6)$ & $47(60.3)$ & $14(73.7)$ & \\
\hline Positive & $38(42.6)$ & $4(44.4)$ & $29(39.7)$ & $5(26.3)$ & 0.543 \\
\hline \multicolumn{6}{|l|}{ Tumor stage, n (\%) } \\
\hline 0 & $2(1.9)$ & $0(0.0)$ & $2(2.6)$ & $0(0.0)$ & \\
\hline $\mathrm{I}$ & $16(15.4)$ & $1(11.1)$ & $12(15.8)$ & $3(15.8)$ & \\
\hline II & $32(30.8)$ & $3(33.3)$ & $21(20.2)$ & $8(42.1)$ & \\
\hline III & $39(37.5)$ & $1(11.1)$ & $33(43.4)$ & $5(26.3)$ & \\
\hline IV & $15(14.4)$ & $4(44.4)$ & $8(10.5)$ & $3(15.8)$ & 0.227 \\
\hline \multicolumn{6}{|c|}{ Distant metastases at diagnosis, n (\%) } \\
\hline Negative & $89(85.6)$ & $5(55.6)$ & $68(89.5)$ & $16(84.2)$ & \\
\hline Positive & $15(14.4)$ & $4(44.4)$ & $8(10.5)$ & $3(15.8)$ & 0.023 \\
\hline \multicolumn{6}{|c|}{ Postoperative chemotherapy, n (\%) } \\
\hline No & $35(33.7)$ & $2(22.2)$ & $26(34.2)$ & $7(36.8)$ & \\
\hline Yes & $69(66.3)$ & $7(77.8)$ & $50(65.8)$ & $12(63.2)$ & 0.719 \\
\hline \multicolumn{6}{|l|}{ Tumor recurrence $^{\mathrm{b}}, \mathrm{n}(\%)$} \\
\hline Negative & $64(71.9)$ & $3(60.0)$ & $46(61.6)$ & $15(93.7)$ & \\
\hline Positive & $25(28.1)$ & $2(40.0)$ & $22(32.4)$ & $1(6.3)$ & 0.093 \\
\hline \multicolumn{6}{|l|}{ MLH1 methylation, $\mathrm{n}(\%)$} \\
\hline- & $94(90.4)$ & $9(100.0)$ & $72(94.7)$ & $13(68.4)$ & \\
\hline+ & $10(9.6)$ & $0(0.0)$ & $4(5.3)$ & $6(31.6)$ & 0.005 \\
\hline
\end{tabular}

${ }^{a}$ Assessed by analysis of variance or Kruskal-Wallis test. ${ }^{\text {bStage } 0-I I I ~ t u m o r s . ~ C I M P, ~ C p G ~ i s l a n d ~ m e t h y l a t o r ~ p h e n o t y p e ; ~ C I M P-L, ~ C I M P-l o w ; ~}$ CIMP-H, CIMP-high; CIMP-N, CIMP-negative, MLH1, MutL homolog 1; NS, not significant.

in Fig. 3A and B. Patients with CIMP-H CRCs exhibited a significantly improved RFS rate compared with those with CIMP-L/N CRCs (Fig. 3B; five-year RFS rate, 93.8 vs. 67.1\%; log-rank test, $\mathrm{P}=0.044)$, although there was no significant difference in OS rate (Fig. 3A; five-year OS rate, 79.0 vs. 68.2\%; $\mathrm{P}=0.383$ ). Cox regression univariate analysis revealed that CIMP-H was a better prognostic indicator for tumor recurrence following curative resection [HR, $0.167 ; 95 \%$ confidence interval (CI), 0.001-0.789], with stage 0-II tumors, an absence of lymphatic and venous invasion and $M L H 1$ methylation as better prognostic factors (Table III). Although the multivariate analysis revealed that tumor stage 0 -II was a significantly better prognostic factor (Table III), the HR of the multivariate analysis for tumor recurrence in CIMP-H tumors was consistently low in stage 0 -II (HR, <0.001; 95\% CI, 0.000-2.281) and in stage III (HR, 0.455; 95\% CI, 0.003-2.228) tumors.

Coexistent lesions within tumors and in the normal mucosae surrounding CRC. In total, 11 (10.6\%) of 104 CRCs presented with neighboring conventional adenoma, but no CRC had 
Table III. Univariate and multivariate analysis of risk factors for recurrence-free survival in stage 0-III colorectal cancer patients.

\begin{tabular}{|c|c|c|c|c|c|c|}
\hline \multirow[b]{2}{*}{ Factor } & \multicolumn{3}{|c|}{ Univariate analysis } & \multicolumn{3}{|c|}{ Multivariate analysis } \\
\hline & HR & $95 \% \mathrm{CI}$ & P-value & HR & $95 \% \mathrm{CI}$ & P-value \\
\hline Age, years $(>60 / \leq 60)$ & 0.639 & $0.287-1.409$ & 0.264 & & & \\
\hline Gender (male/female) & 0.541 & $0.229-1.120$ & 0.132 & & & \\
\hline Tumor location (proximal/distal) & 0.683 & $0.278-1.536$ & 0.364 & & & \\
\hline Tumor size, $\mathrm{mm}(<44 / \geq 44)$ & 0.961 & $0.435-2.134$ & 0.919 & & & \\
\hline Histology (diff/undiff) & 0.872 & $0.302-3.686$ & 0.827 & & & \\
\hline Depth of tumor (T0-2/T3, T4) & 0.416 & $0.098-1.201$ & 0.112 & & & \\
\hline Tumor stage (stage 0-II/III) & 0.247 & $0.096-0.568$ & 0.001 & 0.395 & $0.146-0.969$ & 0.042 \\
\hline $\begin{array}{l}\text { Pathological lymphatic invasion } \\
\text { (negative/positive) }\end{array}$ & 0.448 & $0.189-0.994$ & 0.048 & 0.527 & $0.220-1.187$ & 0.123 \\
\hline $\begin{array}{l}\text { Pathological venous invasion } \\
\text { (negative/positive) }\end{array}$ & 0.305 & $0.135-0.674$ & 0.004 & 0.503 & $0.212-1.156$ & 0.106 \\
\hline $\begin{array}{l}\text { Post-operative chemotherapy } \\
\text { (yes/no) }\end{array}$ & 0.722 & $0.295-1.624$ & 0.440 & & & \\
\hline CIMP status (CIMP-H/L/N) & 0.167 & $0.001-0.789$ & 0.019 & 0.292 & $0.016-1.421$ & 0.149 \\
\hline $\begin{array}{l}\text { MLHI methylation } \\
\text { (positive/negative) }\end{array}$ & $<0.001$ & $0.601-0.601$ & 0.013 & $<0.001$ & $<0.001-1.888$ & 0.141 \\
\hline
\end{tabular}

adjacent serrated lesions. Among the CRCs with adenoma, two were T0, three were $\mathrm{T} 1$, one was $\mathrm{T} 2$ and five were $\mathrm{T} 3$. Four $(21.1 \%)$ out of 19 CIMP-H CRCs presented with coexistent adenomas, together with six $(7.9 \%)$ out of 76 CIMP-L and one $(11.1 \%)$ out of nine CIMP-N CRCs. Two out of four CIMP-H CRCs with adenoma were located in the right colon and the others were in the left colon/rectum; two were T1 and two were T3 tumors. One had MLH1 promoter hypermethylation and the remaining three had no $M L H 1$ hypermethylation. Among the $104 \mathrm{CRC}$ resections, serrated lesions, including five hyperplastic polyps and one serrated adenoma, were present in the normal mucosae around the tumors of four specimens, while conventional adenomas were detected in 25 specimens. In the tumor specimens containing serrated lesions, two CRCs were located in the right colon and two in the left colon/rectum. The serrated lesions were present in one specimen with CIMP-H, in two with CIMP-L and in one with CIMP-N CRC, while adenomatous lesions were distributed in five specimens with CIMP-H, 17 with CIMP-L and three with CIMP-N CRC.

\section{Discussion}

In the present study, according to the number of hypermethylations of 15 promoter CGIs, an almost bimodal distribution of CRCs indicated the presence of the distinct subclass of CRC, termed CIMP-H, which is recognized by an accumulation of hypermethylation of promoter CGIs. In other words, tumor-specific aberrant methylation in promoter CGIs may assemble itself in CIMP-H and randomly occur in remaining CRCs. Although this bimodal distribution in tumors has been demonstrated using several gene marker panels $(5,14)$, Yamashita et al (20) doubted the presence of CIMP, claiming that tumor-specific somatic hypermethylation of six genes (MLH1,pl6, pl4, MGMT, APC and CDH1) was an age-dependent feature and that the distribution of the number of tumors harboring their markers was normal (20). This inconsistency could have been due to the different marker panels used in each study. For instance, APC gene methylation has been inversely linked to classical CIMP-H CRCs (21). Weisenberger et al demonstrated the bimodal distribution of tumors using 14 novel CIMP markers, but the histogram of the methylation frequency of the five classic CIMP markers showed only one peak (5). In the present study, all 15 markers, with the exception of one (CACNAIG), differed from Weisenberger's 14 markers, while the histograms resembled each other. Furthermore, Ogino et al also identified that the CIMP classification error decreased along with an increasing number of markers from one to seven (14). Thus, in addition to the selection of markers, the number of promoter CGIs examined is crucial for the detection of CIMP. The mechanism of this epigenetic instability is unresolved, thus, the definition of CIMP cannot be faultless and depends on the distribution of the methylation frequency of selected markers.

Following the identification of concurrent methylation of several classic CIMP markers in hyperplastic polyposis, large hyperplastic polyps and serrated adenomas $(22,23)$, the serrated lesions, particularly sessile serrated adenomas/polyps, have been described as conceivable precursors of the serrated pathway to CIMP-H CRCs $(16,24)$. In the present study, serrated lesions contiguous with $\mathrm{CRC}$ were not found in any of the CIMP-H tumors. Although this may account for the notion that the ancestor lesion would be replaced by an aggressive successor, $>20 \%$ of CIMP-H CRCs had concomitant 


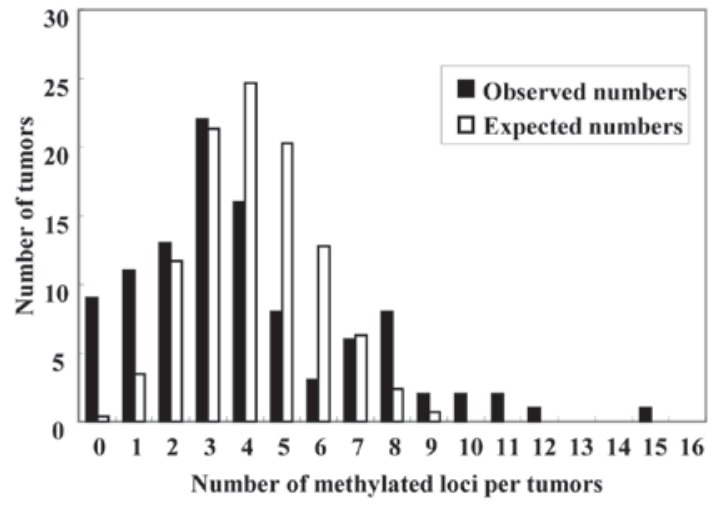

Figure 1. Distribution of the number of tumors and methylated loci per tumor. The expected number is represented by a white bar, and the observed number by a black bar. The observed distribution of carcinomas with each number of aberrantly methylated genes appeared divided into two groups between the high and low methylated groups. With the CpG island methylator phenotype (CIMP) classification used, there was a significant difference between the expected and observed tumor distribution (goodness-of-fit test, $\mathrm{P}<0.001$ ). Carcinomas with methylation of $\geq 7$ of the 15 genes was classified as CIMP-high, methylation of 1-6 genes as CIMP-low, and without any methylation as CIMP-negative.

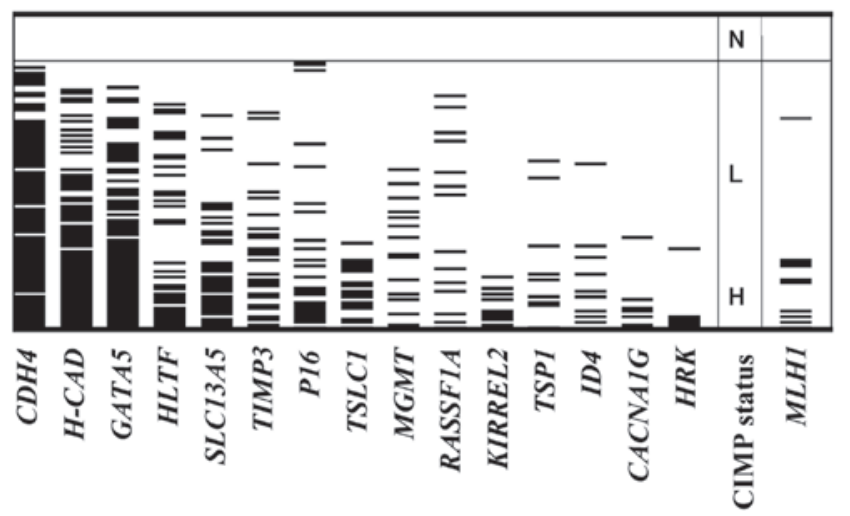

Figure 2. Summary of methylation of 15 promoter $\mathrm{CpG}$ islands in 104 colorectal cancers. Gray bars indicate methylation of each gene. $\mathrm{CpG}$ island methylator phenotype (CIMP) status and MutL homolog 1 (MLHI) methylation shown by gray bars are specified on the right of the methylation data map.

adenomatous lesions that indicated the adenoma-carcinoma pathway of their tumorigenesis. The definition of CIMP-H in the present study was different from the classic or novel CIMP classification, thus the present CIMP-H CRCs may differ from those on the advocated serrated pathway. Jass (25) proposed that one of the molecular subtypes of CRCs that is characterized by CIMP-L, KRAS mutation and microsatellite stable/MSI-L, originates from adenoma or serrated polyps. Although the present study did not examine this molecular discrimination, certain CIMP-H CRCs that have coexistent adenomas can be classified in this subtype. These CIMP-H CRCs may arise from serrated components of mixed hyperplastic and adenomatous polyps, but these polyps are rare. Only one CIMP-H CRC specimen exhibited serrated lesions around the tumor, and each CIMP subtype did not vary in the frequency of serrated lesions in the colonic mucosa around the tumors. Thus, the results failed to support the serrated
A

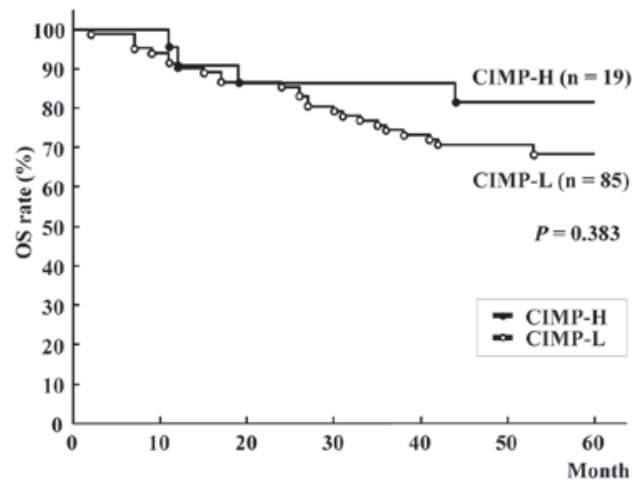

B

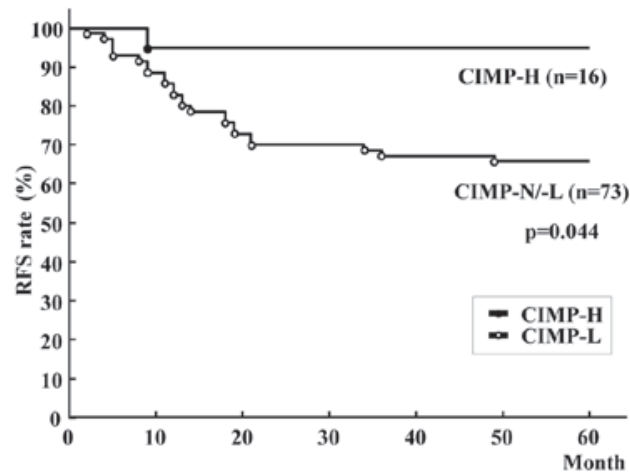

Figure 3. Kaplan-Meier survival curves for (A) OS rate of all patients and (B) RFS of patients following curative resection of colorectal cancer. OS rate was not significantly different between patients with CIMP-H and CIMP-L/-N (five-year OS rate, $79.0 \%$ vs. $68.2 \%$; log-rank test, $\mathrm{P}=0.383$ ), while patients with CIMP-H tumors had better RFS rates than those with CIMP-N/L tumors (five-year RFS rate, 93.8 vs. $67.1 \%$; log-rank test, $\mathrm{P}=0.044$ ). CIMP-H, CpG island methylator phenotype-high; CIMP-L, CIMP-low; CIMP-N, CIMP-negative; RFS, recurrence-free survival; OS, overall survival.

pathway in CIMP-H CRC tumorigenesis, but suggested that a certain fraction of CRCs showing promoter hypermethylation of multiple cancer-related genes is derived from conventional adenoma.

In univariate analysis, the patients with CIMP-H tumors exhibited a significantly improved disease-free survival rate compared with those with CIMP-L/N tumors following curative resection. Similar results have been obtained from a large cohort (4), but this prognostic advantage of CIMP-H is often challenged $(7,8,26)$. Any attempt to involve $M L H 1$ in the CIMP marker panel (14) and to correlate CIMP-H with $B R A F$ mutation (5) would confuse the aforementioned argument further. MLH1 methylation followed by MSI-high (MSI-H) is known to be associated with a good prognosis in patients with sporadic CRC (27-29), while BRAF mutation is one of the genetic markers of a shorter survival time $(4,30)$. Similarly, this controversy over the prognosis of patients with CIMP-H tumors is occasionally explained by the presence of the CIMP subtypes harboring various prognostic features, CIMP-H/MSI-H with frequent $B R A F$ mutation and CIMP-H/MSS with occasional BRAF, but a dominant KRAS mutation (31). Patients with MSI-H CRCs do not benefit from 5-FU-based adjuvant chemotherapy $(32,33)$. Although CIMP-H was associated with $M L H 1$ methylation in the present 
study, $M L H 1$ methylation was not an independent factor for an improved RFS rate. Additionally, 68.4\% of CIMP-H tumors had no $M L H 1$ methylation and $63.2 \%$ of patients with CIMP-H tumors received post-operative 5-FU-based adjuvant chemotherapy in the present study. Thus, the improved RFS rates of patients with CIMP-H tumors was not solely a result of MSI-H following $M L H 1$ methylation. Iacopetta et al demonstrated that CIMP-H was a predictor of the survival benefit from 5-FU-based chemotherapy in CRC patients (9). Therefore, this prognostic advantage of patients with CIMP-H CRCs could be augmented by 5-FU-based adjuvant chemotherapy. However, 5-FU-based adjuvant chemotherapy was recently shown not to improve, but to worsen the disease-free survival of patients with stage II or III CIMP-positive CRC (34). The criteria of CIMP, the proportion of MLH methylation in CIMP-H tumors and the number of patients receiving adjuvant chemotherapy largely differed between these studies. Thus, a large cohort study based on universal CIMP consent using coherent CIMP markers is required to resolve this critical issue.

Little is known about the CRCs that are without methylation of any promoter CGIs; named CIMP-N in the present study. The absence of aberrant methylation of any promoter CGIs in these patients confers possible global hypomethylation, which has been often associated with chromosomal instability in CRC $(35,36)$. Instead, an inverse association between CIMP-H and chromosomal instability has been shown $(37,38)$. Similar to the association between the shorter survival time and LINE-1 hypomethylation among CRC patients (39), frequent metastases at the time of diagnosis in patients with CIMP-N CRC suggest that CIMP-N tumors are more aggressive than CIMP-L/H tumors. In the present study, the histogram of the CIMP-L/N CRCs was almost a Gaussian distribution and the number of tumors in this subset was small, therefore, the meaning of this phenotype in CRCs remains uncertain.

There were certain limitations to this study. Firstly, $B R A F$ and $K R A S$ were not sequenced. Although the $B R A F$ mutation is one of the genetic traits of CIMP-H CRCs, the BRAF mutation in CIMP has varied in each study, ranging from $21.6 \%$ using a classic CIMP marker set (26), to $73 \%$ using novel markers (5). The mechanism connecting the $B R A F$ mutation and aberrant methylation of promoter CGIs remains unclear, and the importance of this mutation to predict prognosis was not proven in recent larger studies involving $>1,000 \mathrm{CRC}$ patients $(27,40)$. Additionally, MSI status was not examined in the present study. Finally, this was a single-institution retrospective study, and the numbers of patients and genes examined were not sufficient to allow definitive conclusions.

The panel of promoter CGIs in this study included KIRREL2 and SLC13A5, which have previously been identified as the differentially-methylated CGIs in pancreatic cancer and cloned using methylated $\mathrm{CpG}$ island amplification-representational difference analysis from pancreatic cancer cell lines (18). Cancer-specific methylation of these CGIs, loss of expression of these genes in CRC cell lines that had hypermethylation of these promoter CGIs and restoration of their expression by 5-aza-2-deoxycytidine treatment (data not shown) suggest possible involvement of promoter methylation of these genes in colorectal carcinogenesis. For example, SLC13A5, a member of the solute carrier (SLC) families and a $\mathrm{Na}^{+} /$sulfate/selenate/thiosulfate/carboxylate symporter (41), is one of the hallmarks of
CIMP in renal cell carcinoma (42). SLC13A5 is differentially methylated between glioblastoma and normal brain tissue, as shown by whole-genome integrative analysis (43). Certain SLC family members increase chemosensitivity against anticancer drugs by mediating the cellular uptake of hydrophilic drugs (44). One of the sodium transporter families also has tumor suppressor activity, and aberrant methylation of promoter CGI is detected in aberrant crypt foci, which is considered to be the initial lesion of the serrated adenoma-carcinoma pathway (45). Thus, future studies on the novel target gene for aberrant promoter methylation would shed light on our understanding of cancer epigenetics and the carcinogenesis of CRC.

\section{References}

1. Shen L and Issa JP: Epigenetics in colorectal cancer. Curr Opin Gastroenterol 18: 68-73, 2002.

2. Toyota M, Ahuja N, Ohe-Toyota M, et al: $\mathrm{CpG}$ island methylator phenotype in colorectal cancer. Proc Natl Acad Sci USA 96: 8681-8686, 1999.

3. Hawkins N, Norrie M, Cheong K, et al: $\mathrm{CpG}$ island methylation in sporadic colorectal cancers and its relationship to microsatellite instability. Gastroenterology 122: 1376-1387, 2002.

4. Ogino S, Nosho K, Kirkner GJ, et al: CpG island methylator phenotype, microsatellite instability, BRAF mutation and clinical outcome in colon cancer. Gut 58: 90-96, 2009.

5. Weisenberger DJ, Siegmund KD, Campan M, et al: CpG island methylator phenotype underlies sporadic microsatellite instability and is tightly associated with BRAF mutation in colorectal cancer. Nat Genet 38: 787-793, 2006.

6. Shen L, Toyota M, Kondo Y, et al: Integrated genetic and epigenetic analysis identifies three different subclasses of colon cancer. Proc Natl Acad Sci USA 104: 18654-18659, 2007.

7. Ahn JB, Chung WB, Maeda O, et al: DNA methylation predicts recurrence from resected stage III proximal colon cancer. Cancer 117: 1847-1854, 2011.

8. Van Rijnsoever M, Grieu F, Elsaleh H, et al: Characterisation of colorectal cancers showing hypermethylation at multiple $\mathrm{CpG}$ islands. Gut 51: 797-802, 2002.

9. Iacopetta B, Kawakami K and Watanabe T: Predicting clinical outcome of 5-fluorouracil-based chemotherapy for colon cancer patients: is the $\mathrm{CpG}$ island methylator phenotype the 5-fluorouracil-responsive subgroup? Int J Clin Oncol 13: 498-503, 2008.

10. Shen L, Catalano PJ, Benson AB III, et al: Association between DNA methylation and shortened survival in patients with advanced colorectal cancer treated with 5-fluorouracil based chemotherapy. Clin Cancer Res 13: 6093-6098, 2007.

11. Van Rijnsoever M, Elsaleh H, Joseph D, et al: CpG island methylator phenotype is an independent predictor of survival benefit from 5-fluorouracil in stage III colorectal cancer. Clin Cancer Res 9: 2898-2903, 2003.

12. Arnold CN, Goel A, Compton C, et al: Evaluation of microsatellite instability, hMLH1 expression and hMLH1 promoter hypermethylation in defining the MSI phenotype of colorectal cancer. Cancer Biol Ther 3: 73-78, 2004.

13. Samowitz WS, Albertsen H, Herrick J, et al: Evaluation of a large, population-based sample supports a $\mathrm{CpG}$ island methylator phenotype in colon cancer. Gastroenterology 129: 837-845, 2005.

14. Ogino S, Kawasaki T, Kirkner GJ, et al: Evaluation of markers for $\mathrm{CpG}$ island methylator phenotype (CIMP) in colorectal cancer by a large population-based sample. J Mol Diagn 9: 305-314, 2007.

15. Hawkins NJ and Ward RL: Sporadic colorectal cancers with microsatellite instability and their possible origin in hyperplastic polyps and serrated adenomas. J Natl Cancer Inst 93: 1307-1313, 2001.

16. Gaiser T, Meinhardt S, Hirsch D, et al: Molecular patterns in the evolution of serrated lesion of the colorectum. Int J Cancer 132: 1800-1810, 2013

17. George SM, Mäkinen MJ, Jernvall P, et al: Classification of advanced colorectal carcinomas by tumor edge morphology: evidence for different pathogenesis and significance of polypoid and nonpolypoid tumors. Cancer 89: 1901-1909, 2000.

18. Ueki T, Toyota M, Skinner H, et al: Identification and characterization of differentially methylated $\mathrm{CpG}$ islands in pancreatic carcinoma. Cancer Res 61: 8540-8546 2001. 
19. Herman JG, Graff JR, Myöhänen S, et al: Methylation-specific PCR: a novel PCR assay for methylation status of $\mathrm{CpG}$ islands. Proc Natl Acad Sci USA 93: 9821-9826, 1996.

20. Yamashita K, Dai T, Dai Y, et al: Genetics supersedes epigenetics in colon cancer phenotype. Cancer Cell 4: 121-131, 2003.

21. Iacopetta B, Grieu F, Li W, et al: APC gene methylation is inversely correlated with features of the $\mathrm{CpG}$ island methylator phenotype in colorectal cancer. Int J Cancer 119: 2272-2278, 2006.

22. Chan AO, Issa JP, Morris JS, et al: Concordant CpG island methylation in hyperplastic polyposis. Am J Pathol 160: 529-536, 2002.

23. Park SJ, Rashid A, Lee JH, et al: Frequent $\mathrm{CpG}$ island methylation in serrated adenomas of the colorectum. Am J Pathol 162: 815-822, 2003.

24. Young J and Jass JR: The case for a genetic predisposition to serrated neoplasia in the colorectum: hypothesis and review of the literature. Cancer Epidemiol Biomarkers Prev 15: 1778-1784, 2006.

25. Jass JR: Classification of colorectal cancer based on correlation of clinical, morphological and molecular features. Histopathology 50: 113-130, 2007.

26. Barault L, Charon-Barra C, Jooste V, et al: Hypermethylator phenotype in sporadic colon cancer: study on a population-based series of 582 cases. Cancer Res 68: 8541-8546, 2008.

27. Hutchins G, Southward K, Handley K, et al: Value of mismatch repair, KRAS, and BRAF mutations in predicting recurrence and benefits from chemotherapy in colorectal cancer. J Clin Oncol 29: 1261-1270, 2011.

28. Popat S, Hubner R and Houlston RS: Systematic review of microsatellite instability and colorectal cancer prognosis. J Clin Oncol 23: 609-618, 2005.

29. Sinicrope FA, Foster NR, Thibodeau SN, et al: DNA mismatch repair status and colon cancer recurrence and survival in clinical trials of 5-fluorouracil-based adjuvant therapy. J Natl Cancer Inst 103: 863-875, 2011.

30. Samowitz WS, Sweeney C, Herrick J, et al: Poor survival associated with the BRAF V600E mutation in microsatellite-stable colon cancers. Cancer Res 65: 6063-6069, 2005.

31. Ang PW, Loh M, Liem N, et al: Comprehensive profiling of DNA methylation in colorectal cancer reveals subgroups with distinct clinicopathological and molecular features. BMC Cancer 10: 227, 2010

32. Carethers JM, Smith EJ, Behling CA, et al: Use of 5-fluorouracil and survival in patients with microsatellite-unstable colorectal cancer. Gastroenterology 126: 394-401, 2004.
33. Ribic CM, Sargent DJ, Moore MJ, et al: Tumor microsatellite-instability status as a predictor of benefit from fluorouracil-based adjuvant chemotherapy for colon cancer. N Engl J Med 349: 247-57, 2003.

34. Jover R, Nguyen TP, Perez-Carbonell L, et al: 5-Fluorouracil adjuvant chemotherapy does not increase survival in patients with $\mathrm{CpG}$ island methylator phenotype colorectal cancer. Gastroenterology 140: 1174-1181, 2011.

35. Matsuzaki K, Deng G, Tanaka H, et al: The relationship between global methylation level, loss of heterozygosity, and microsatellite instability in sporadic colorectal cancer. Clin Cancer Res 11: 8564-8569, 2005.

36. Rodriguez J, Frigola J, Vendrell E, et al: Chromosomal instability correlates with genome-wide DNA demethylation in human primary colorectal cancers. Cancer Res 66: 8462-8468, 2006.

37. Derks S, Postma C, Carvalho B, et al: Integrated analysis of chromosomal, microsatellite and epigenetic instability in colorectal cancer identifies specific associations between promoter methylation of pivotal tumour suppressor and DNA repair genes and specific chromosomal alterations. Carcinogenesis 29: 434-439, 2008.

38. Goel A, Nagasaka T, Arnold CN, et al: The $\mathrm{CpG}$ island methylator phenotype and chromosomal instability are inversely correlated in sporadic colorectal cancer. Gastroenterology 132: 127-138, 2007.

39. Ogino S, Nosho K, Kirkner GJ, et al: A cohort study of tumoral LINE-1 hypomethylation and prognosis in colon cancer. J Natl Cancer Inst 100: 1734-1738, 2008.

40. Roth AD, Tejpar S, Delorenzi M, et al: Prognostic role of KRAS and BRAF in stage II and III resected colon cancer: results of the translational study on the PETACC-3,EORTC 40993, SAKK 60-00 trial. J Clin Oncol 28: 466-474, 2010.

41. He L, Vasiliou K and Nebert DW: Analysis and update of the human solute carrier (SLC) gene superfamily. Hum Genomics 3: 195-206, 2009.

42. Arai E, Chiku S, Mori T, et al: Single-CpG-resolution methylome analysis identifies clinicopathologically aggressive $\mathrm{CpG}$ island methylator phenotype clear cell renal cell carcinomas. Carcinogenesis 33: 1487-1493, 2012.

43. Etcheverry A, Aubry M, de Tayrac M, et al: DNA methylation in glioblastoma: impact on gene expression and clinical outcome. BMC genomics 11: 701, 2010.

44. Huang Y and Sadée W: Membrane transporters and channels in chemoresistance and -sensitivity of tumor cells. Cancer Lett 239: 168-182, 2006.

45. Li H, Myeroff L, Smiraglia D, et al: SLC5A8, a sodium transporter, is a tumor suppressor gene silenced by methylation in human colon aberrant crypt foci and cancers. Proc Natl Acad Sci USA 100: 8412-8417, 2003 